\title{
Development of space environment customized risk estimation for satellites (SECURES)
}

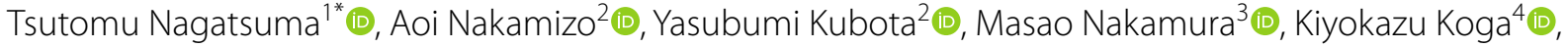 \\ Yoshizumi Miyoshi ${ }^{5}$ [D and Haruhisa Matsumoto ${ }^{4}$
}

\begin{abstract}
Plasma variations in the geospace environment driven by solar wind-magnetosphere interactions are one of the major causes of satellite anomaly. To mitigate the effect of satellite anomaly, the risk of space weather disturbances predicted by space weather forecasting needs to be known in advance. However, the risk of satellite anomaly owing to space weather disturbances is not the same for all satellites, because the risk depends not only on the space environment itself but also on the design and materials of individual satellites. From the viewpoint of satellite operators, it is difficult to apply a general alert level of the space environment to the risk of individual satellites. To provide tailored space weather information, we have developed SECURES (Space Environment Customized Risk Estimation for Satellites) by combining models of the space environment and those of spacecraft charging. In SECURES, we focus on the risk of spacecraft charging (surface/internal) for geosynchronous satellites. For the risk estimation of surface charging, we have combined the global magnetosphere magnetohydrodynamics (MHD) model with the satellite surface charging models. For the risk estimation of internal charging, we have combined the radiation belt models with the satellite internal charging models. We have developed prototype products for both types of charging/electrostatic discharge (ESD). The development of SECURES and our achievements are introduced in this paper.
\end{abstract}

Keywords: Space weather forecasting, Geospace, Satellite anomaly, Satellite charging, Customized risk estimation

\section{Introduction}

The space around Earth (geospace) has been used by many types of satellite for various purposes, such as telecommunications, broadcasting, Earth observation, and positioning. The plasma and electromagnetic environments in geospace change significantly owing to geospace disturbances, such as substorms and storms caused by solar wind-magnetosphere interactions. They can also be affected by high-energy particles, known as solar energetic particle (SEP) events. The geospace disturbances cause various satellite anomalies, such as charging/discharging and central processing unit (CPU)

\footnotetext{
*Correspondence: tnagatsu@nict.go.jp

${ }^{1}$ National Institute of Information and Communications Technology,

2-1-3 Katahira, Aoba-ku, Sendai, Miyagi 980-0812, Japan

Full list of author information is available at the end of the article
}

malfunctions. Therefore, satellites are designed and developed with measures to prevent anomalies due to geospace disturbances during operation and in the satellite's orbit.

A space weather forecast provides the current status and future condition of the geospace environment on the basis of observation and modeling. If there is a possibility of spacecraft anomalies due to the space environment, the following three utilizations of space weather forecasts are necessary to improve the stability and safety of satellite operation.

Firstly, when a satellite anomaly occurs, triage determination should be the first action. The satellite operator needs to identify the cause of the anomaly (misoperation, manufacturing problem, or space environment) to decide the next action for mitigating the effect of the anomaly. 
For this purpose, it is necessary to understand the current condition (nowcast) of the space environment around the satellite to determine whether the malfunction/failure is caused by the space environment or other factors (O’Brien et al. 2013).

Secondly, if there is a high possibility that the satellite anomaly is caused by the space environment, postanalysis of data will be performed to clarify the relationship between geospace disturbances and the satellite anomaly. It is necessary to take future measures and to improve the satellite itself on the basis of the outcome of the postanalysis of data analysis.

Thirdly, in the case of conducting critical operations, such as attitude control and/or rewriting onboard programs, where mistakes and errors can seriously affect the satellite, the satellite operator should refer to the space weather forecast and make a Go/NoGo decision for critical operations in order to reduce the risk of satellite anomaly. In addition, if the risk of satellite anomaly due to space weather disturbance can be predicted, the stability and continuity of satellite operation can be improved by taking measures, such as increasing the number of staff in charge of operation and preparing backup plans. It is possible to realize rapid recovery in the event of a satellite anomaly.

However, the specific utilization of space weather forecasts in the current satellite operation is still in the development stage for the following reasons. First, there are many unsolved problems regarding the relationship between space weather disturbances and satellite anomalies. Since the operating satellite is in space, the status of the satellite cannot be directly confirmed, making it difficult to directly investigate the cause of any failure. In addition, it is difficult for satellite manufacturers and satellite operators to disclose detailed information about satellite anomalies to the public. To overcome these problems, the United Nations Committee on the Peaceful Uses of Outer Space (UN/COPUOS) has called for the sharing of information about satellite design standards, space weather observations and models implemented in each country, satellite anomalies, and efforts to mitigate the effects of satellite anomalies (UN/COPUOS 2019).

Another issue is the accuracy and lead time of space weather forecasts. The space environment significantly changes in space and time depending on the solar windmagnetosphere interactions. Therefore, the condition of the surrounding space environment may be considerably different depending on the position of the satellite, and the space weather forecast is required to reproduce the difference. In addition, since the conditions of the geospace environment are driven by solar wind, forecasts with sufficient lead times cannot be realized unless the state of solar wind can be predicted in advance. However, since there are few observations between the Sun-Earth line, the current status of forecasting lead time is about $1 \mathrm{~h}$ ahead when using data from the solar wind observation at the L1 point of the Sun-Earth system. Prediction with longer lead times is much less accurate.

Finally, as will be described later, the risk of satellite anomaly due to the geospace environment also varies depending on the materials and structure used for the satellite itself. In other words, it is difficult for satellite operators to judge the risk of individual satellites from only the space weather forecasts. Therefore, to achieve the safety and stability of satellite operation, it is necessary to understand not only the status of the geospace environment but also the risks of individual satellites.

On the basis of this background, we have been developing a charging risk estimation scheme for satellite operators, called SECURES (Space Environment Customized Risk Estimation for Satellites), under PSTEP (Project for Solar-Terrestrial Environment Prediction) (Nagatsuma 2017). In this paper, we will introduce the outline of SECURES, the surface charging module, and the internal charging module, and summarize and discuss our achievements.

\section{Outline of SECURES}

Electrostatic discharge (ESD) due to space weather disturbances is one of the major causes of satellite anomalies (e.g., Ferguson et al. 2015). More than half of the satellite anomalies are caused by ESD (Koons et al. 1999). There are two types of charging mechanism that will cause ESD, namely, surface charging and internal charging. Surface charging occurs on the surface of the satellite structure and is caused by exposure to plasma of several tens to several hundred $\mathrm{keV}$. An electric potential is formed on the surface of the satellite by the inflow of ions and electrons from the plasma environment around the satellite and the emission of photoelectrons because of sunlight. Internal charging occurs inside the satellite structure. Electrons with energies higher than several hundred $\mathrm{keV}$ penetrate the wall of the satellite structure as well as the interior, leading to the charging and discharging of cables and equipment inside the satellite. Owing to plasma variations in geospace, the electric potential on the surface and inside of the satellite may change, resulting in electrical discharges that lead to malfunctions and failures. Charging and discharging conditions differ depending on the satellite shape and materials even if the space environment condition is the same. Therefore, it is difficult for a satellite operator to identify the operational risk of an individual satellite using only the general information of a space weather nowcast/forecast. This problem will be reduced by estimating the charging risk of 
an individual satellite using satellite charging models while providing the current and future conditions of the space environment at the position of an individual satellite using models of the space weather forecast.

Figure 1 shows the concept of SECURES. Specifically, for each of surface and internal charging, the predicted physical parameters in the space environment (electron/ ion temperature, density, etc.) at the position of the individual satellite and the structure and material model of the satellite are used as input of the satellite charging model to estimate the risk of charging.

The space environment that causes surface charging is simulated using the Global Magnetohydrodynamics (MHD) model. The information on the simulated space environment is used to calculate the charging of an individual satellite under a certain space environment condition using a satellite charging model, such as the Spacecraft Plasma Interaction System (SPIS) (Roussel et al. 2008) or Multi-Utility Spacecraft Charging Analysis Tool (MUSCAT) (Hosoda et al. 2008; Muranaka et al. 2008). High-energy particles that cause internal charging are predicted by radiation belt simulation. The information on the predicted space environment is used to calculate the internal charging with MUSCAT. Detailed information on the surface charging and internal charging parts of SECURES will be introduced in the following sections.

\section{Surface charging part of SECURES}

Toward the surface charging/ESD risk assessment, we combine a surface charging analysis model (SPIS or MUSCAT), which estimate spacecraft potentials on the basis of a predesigned satellite model, including information on the shape and materials of the satellite, and a global magnetosphere MHD model, which simulates the plasma environment for the input of the charging analysis model. We have developed a prototype surface charging/ ESD risk assessment system targeting the geostationary earth orbit (GEO) region. In this section, the global magnetosphere MHD model, charging analysis model, and the overview of the prototype surface charging/ESD risk assessment system are described.

\section{Plasma environment at GEO estimated using the real-time} global magnetosphere MHD model

The global magnetosphere MHD model used in the surface charging part of SECURES was originally developed by Tanaka (1994) and Tanaka et al. (2010). The message passing interface (MPI) parallelized version of the model is called the REPPU (REProduce Plasma Universe) code

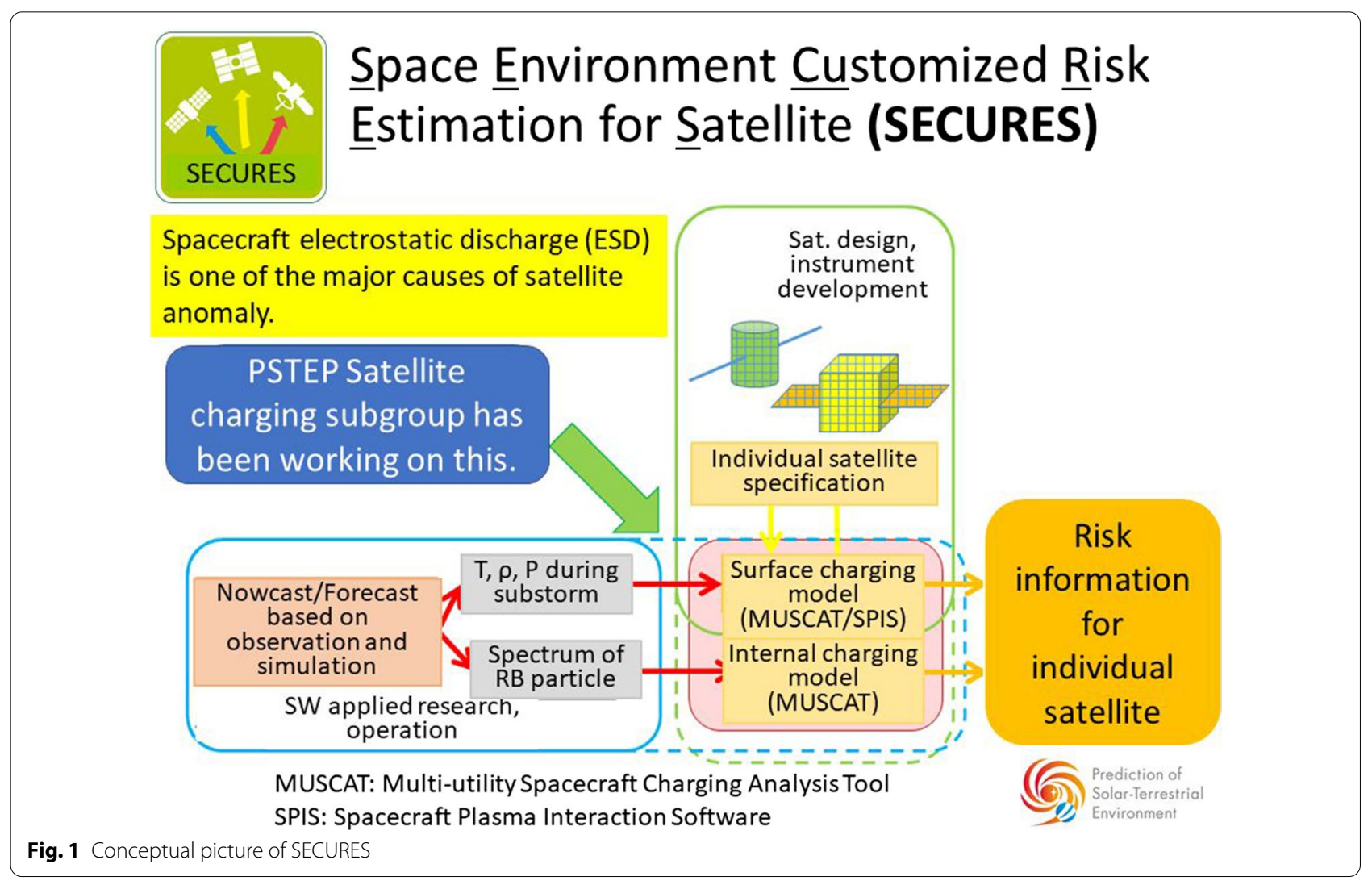


(Tanaka 2015). The model is characterized by a triangular unstructured grid system (Nakamizo et al. 2009), which enables us to simulate global systems, including fine structures in their center with sufficiently high spatial resolution and numerical stability. It is shown that the code is sufficiently robust to simulate magnetospheric responses to extreme conditions, such as very high solar wind velocity and density and a high intensity of the interplanetary magnetic field (Kubota et al. 2017). The relationships among the space environment data and surface charging data obtained from the Michibiki-1 satellite and the space environment data obtained from global magnetosphere MHD simulation are examined as a first step (Nagatsuma et al. 2018).

For simplicity, the original code assumes that Earth's magnetic dipole axis and the rotation axis coincide with each other. Therefore, it does not meet the requirements for practical use and operation as it is, because in the actual solar-terrestrial system, Earth's rotation axis is inclined with respect to the ecliptic plane, and the magnetic dipole axis is tilted from the rotation axis, completing the precession.

To perform a more realistic simulation, we have improved the model by introducing the inclination and precession of the magnetic dipole axis (Kubota et al. 2019). With this improved model, a real-time global magnetosphere simulation on the High-Performance Computing System at the National Institute of Information and Communications Technology (NICT) is carried out. The real-time simulation is driven by the real-time solar wind data at the L1 point of the Sun-Earth system provided by the National Oceanic and Atmospheric Administration's Space Weather Prediction Center (NOAA/ SWPC). Because the average propagation time of the solar wind from the L1 point to the front of Earth's magnetosphere is about $1 \mathrm{~h}$, and the processing time of the real-time simulation system is about $20 \mathrm{~min}$, the total lead time of the real-time simulation is about $40 \mathrm{~min}$ on average. The outputs of the simulation are shown in a web page as a real-time product. Figure 2 shows a screenshot of the web page which shows input parameters and the real-time simulation result. Currently, the realtime product is used internally for the operational space weather forecast in Japan.

The spacecraft charging analysis model, which will be introduced in the next subsection, requires the densities and temperatures of ions and electrons $\left(N_{i}, N_{e}, T_{i}\right.$, and $T_{e}$ ) as the input parameters of the model. Since MHD models cannot simulate these parameters in principle, the following empirical scheme is introduced for estimating them using the MHD model.

Nakamura (2012) statistically compared the plasma parameters in GEO simulated using the old version of our global magnetosphere MHD model and those obtained from the Magnetospheric Plasma Analyzer (MPA) onboard Los Alamos National Laboratory (LANL) satellites. He showed that the simulated MHD pressure $\mathrm{P}_{\text {sim }}$ in the nightside GEO region has a good correlation with the observed electron pressure. We have applied the same approach as Nakamura (2012) to the current version of our MHD model. We used the plasma moment data of LANL/MPA from February to April 2006 available via the Coordinated Data Analysis Web (CDAWeb). First, we searched for events in which LANL/MPA observed negative potential in association with the occurrence of isolated substorms while the satellites were on the nightside. A total of 12 events were selected. For each event, we performed MHD simulations using the OMNI solar wind and IMF data (available via CDAWeb) as the input. We extracted the time series of plasma parameters along the satellite orbits from the simulation data and then compared them with the LANL/MPA data.

Figure 3 shows an example of the deep negative satellite charging event during an isolated substorm that occurred at around 10:00 UT on February 15, 2006. The top three panels show the OMNI data: the three components of IMF in the GSM coordinate system, solar wind speed, and solar wind density. The fourth and fifth panels show the cross polar cap potential (CPCP) estimated from the PCN index (Troshichev et al. 1996) and AU/AL indices, respectively. The $\mathrm{CPCP}$ and AU/AL indices deduced from the simulation are shown by the red lines in each panel. The next three panels show plasma moment data. The observed electron, ion, and simulation data are shown by the solid black, dotted black, and solid red lines, respectively. Shown from the sixth to eighth panels are, respectively, $N_{e}, N_{i}$, and simulated plasma density $N_{\text {sim }} ; T_{e}, T_{i}$, and simulated plasma temperature $T_{\text {sim }}$; and $P_{e}, P_{i}$, and simulated plasma pressure $P_{\text {sim }}$. The ninth panel shows the satellite potential. The bottom two panels show the satellite orbit in (MLT, L) and in $(X, Y, Z)$ of the GSM coordinate system, respectively. Around the period of the substorm, the LANL satellite, which remained in the midnight sector, observed the enhancements of electron and ion parameters, leading to a deep negative spacecraft potential that reached about $-6000[\mathrm{~V}]$.

Similar to the result obtained by Nakamura (2012), $P_{\text {sim }}$ is well correlated with the observed $P_{e}$. This characteristic is confirmed for all selected events. On the basis of this result, we consider a method for estimating $N_{e}$ and $T_{e}$ from the simulation data.

For each selected event, we searched for the simulation time when $P_{\text {sim }}$ peaked within \pm 30 min from the time when $P_{e}$ peaked, leading to negative charging, and we extracted simulation data and observational data at each timing of the pressure peak. Figure 4 shows the scatter 


\section{Real-time Global Magnetosphere Simulation}

\section{by improved version of REPPU code}

Caution!! This plots can be used for quick look only. DSCOVR solar wind data is provided by NOAA/SWPC
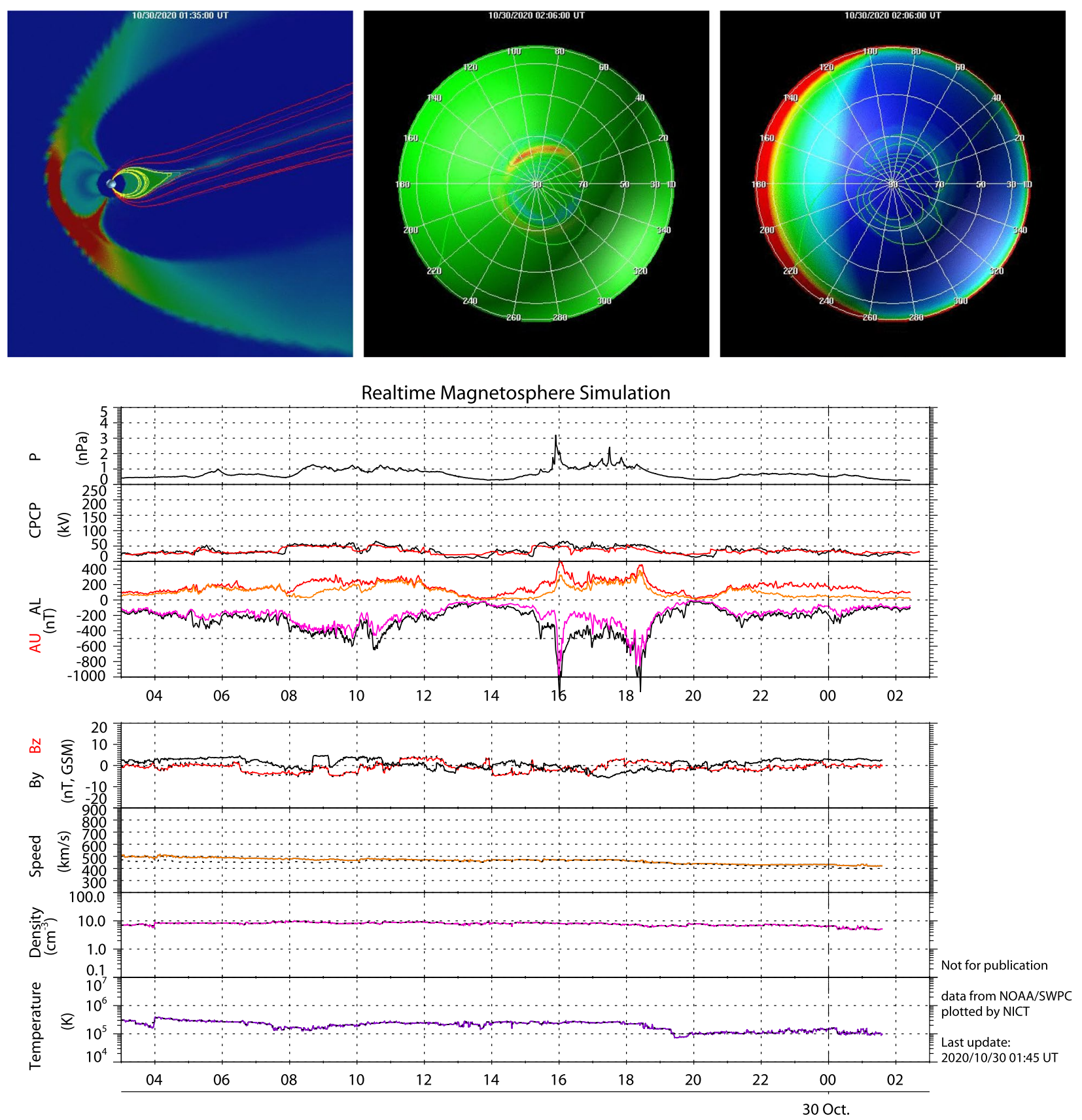

Fig. 2 A screenshot of a web page which shows input parameters and the real-time global magnetosphere MHD simulation result

plots of extracted data. Figure $4 \mathrm{a}-\mathrm{c}$ shows the scatter plots of $N_{e}$ versus $N_{\text {sim }}, T_{e}$ versus $T_{\text {sim }}$, and $P_{e}$ versus $P_{\text {sim }}$, respectively. Figure $4 \mathrm{~d}$ shows the scatter plot of observed $T_{i}$ versus $T_{e}$. $P_{\text {sim }}$ has a good correlation with $P_{e}$. On the other hand, $N_{\text {sim }}$ tends to be much higher than $N_{e}$, and $T_{\text {sim }}$ tends to be lower than $T_{e}$. We focus on the result that the observed $N_{e}$ is about $1 / \mathrm{cm}^{3}$. Because $P_{\text {sim }}$ is in good agreement with $P_{e}$, and $N_{e}$ is almost $1 / \mathrm{cm}^{3}$, we derive 


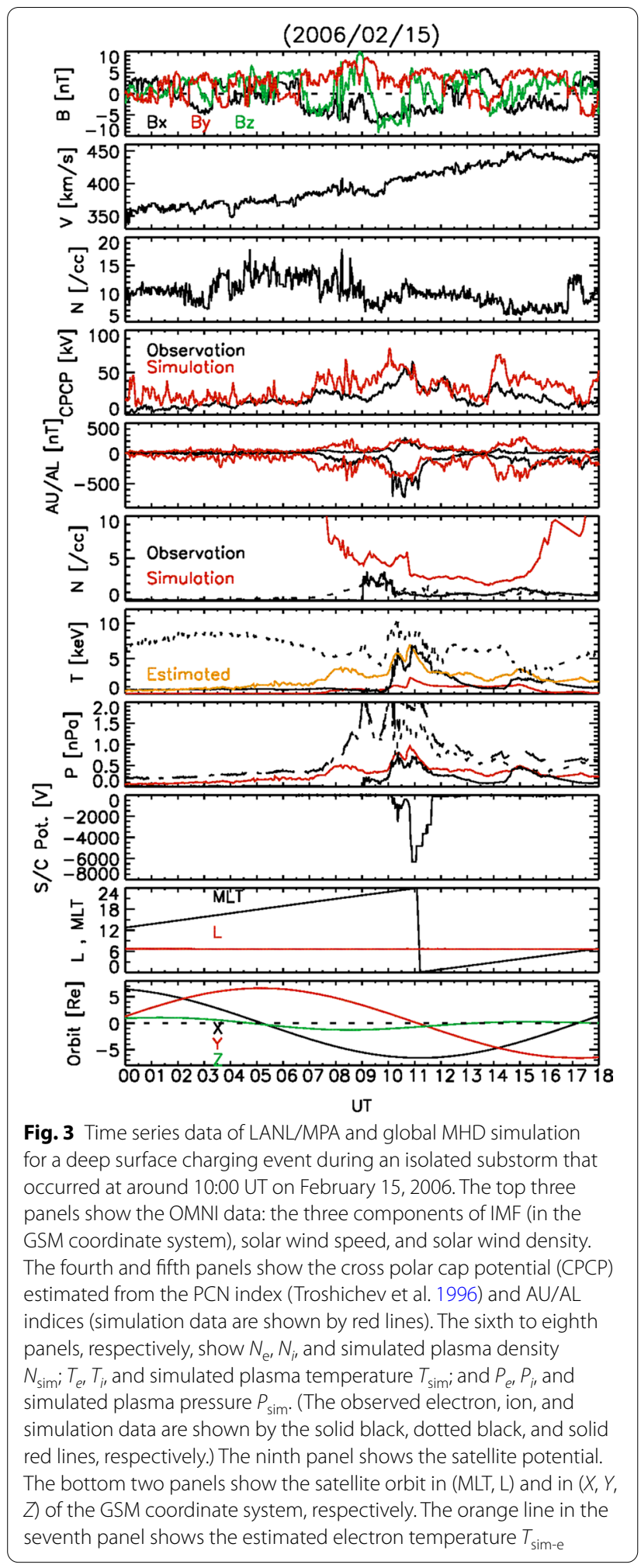

$T_{e}$ from $T_{\text {sim }}$ or $P_{\text {sim }}$ assuming $N_{e}=1$. The electron temperature $\underline{T}_{\text {sim-e }}$ estimated in this way is plotted in Fig. $4 \mathrm{~b}$ by orange asterisks. Also, in Fig. 3 , we plot $T_{\text {sim-e }}$ by the orange line; $T_{\text {sim-e }}$ well traces $T_{e}$, better than $T_{\text {sim }}$ itself.

More sophisticated estimation methods may be conceivable. However, we adopt the simplified method at present. To obtain $T_{i}$, we multiply the derived $T_{\text {sim-e }}$ by a factor of 1.9. This factor is the average ratio of $T_{i}$ to $T_{e}$ in Fig. 4d. On the basis of these empirical relationships, $N_{i}$, $T_{i}, N_{e}$, and $T_{e}$ from the real-time simulation data for the nightside GEO region are estimated.

\section{Estimating real-time risk of surface charging using SPIS}

The surface charging of an individual satellite is calculated using SPIS under space environment conditions and the densities and temperatures of ions and electrons $\left(N_{i}\right.$, $N_{e}, T_{i}$, and $\left.T_{e}\right)$. In our prototype product, two examples of geometric models are introduced as a test case of our risk estimation. One is Van Allen Probes, a typical spin-stabilized scientific satellite, the surfaces of which are conductive and electrically connected (Stratton et al. 2013). The other is the Michibiki-1 satellite, a typical three-axis stabilized geosynchronous satellite with dielectric materials (Inaba et al. 2009). Figure 5 shows geometric models of the Van Allen Probes and the Michibiki-1 satellite used in the surface charging calculation. We calculate the absolute charging potential for the geometric model of Van Allen Probes. The absolute charging would have an effect on some scientific observations. We calculate the floating potentials of the surface materials and the local differential charging potentials for the geometric model of the Michibiki-1 satellite. The high differential charging potential results in ESD on the satellite surface and may induce spacecraft anomalies. Therefore, the estimation of the differential charging potentials is important for surface charging/ESD risk assessment.

However, it is impossible to obtain the surface potentials in near real time from real-time global magnetosphere MHD simulation data because the charging calculation of the Michibiki-1 satellite model using SPIS takes hours or days for one space environment condition. Therefore, we have developed a quick method of estimating the equilibrium surface potential using precalculated results. The estimation of the surface potential for the environment parameters $N_{i}, T_{i}, N_{e}$, and $T_{e}$ as four independent variables is very complicated. However, as shown in the previous subsection, we can calculate the surface charging potentials for the conditions $N_{e}=N_{i}=1 /$ 


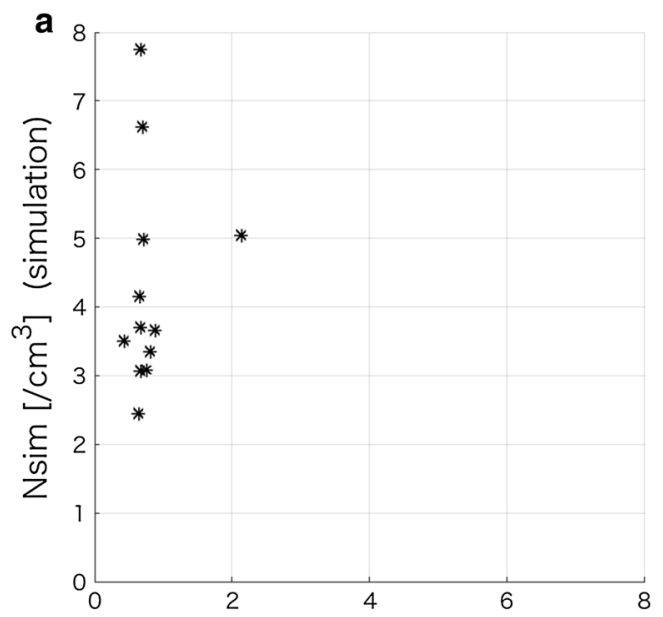

$\mathrm{Ne}\left[/ \mathrm{cm}^{3}\right]$ (observation)

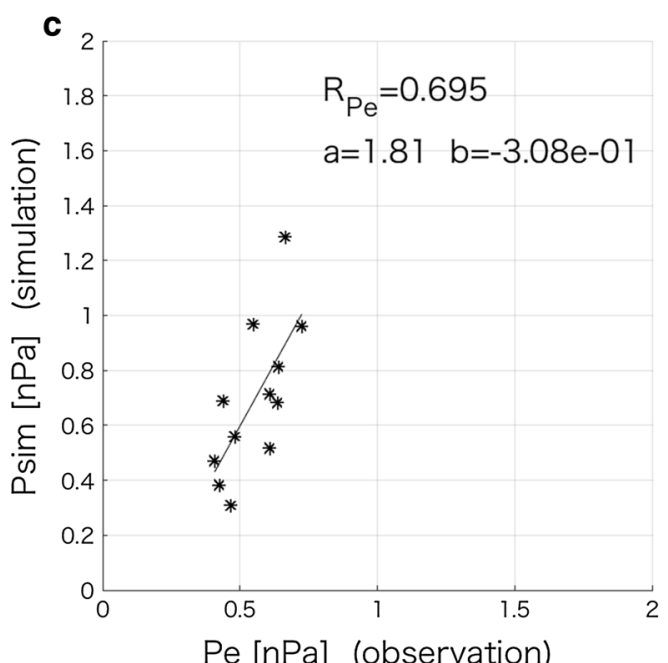

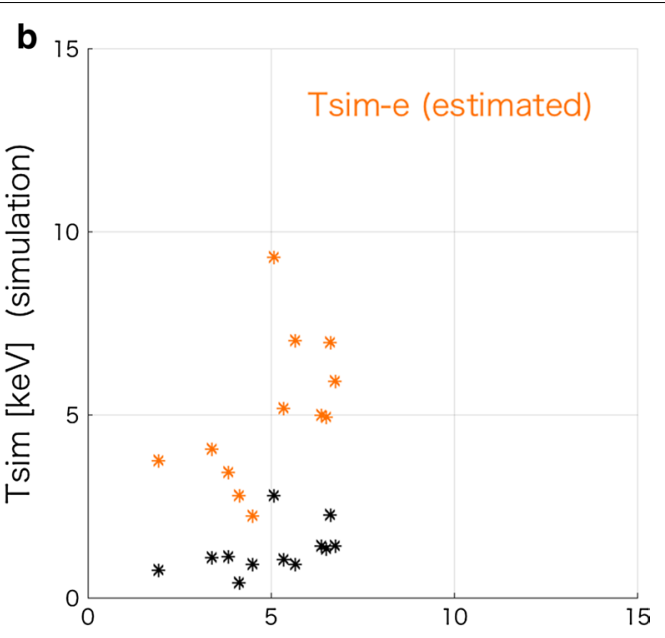

Te [keV] (observation)

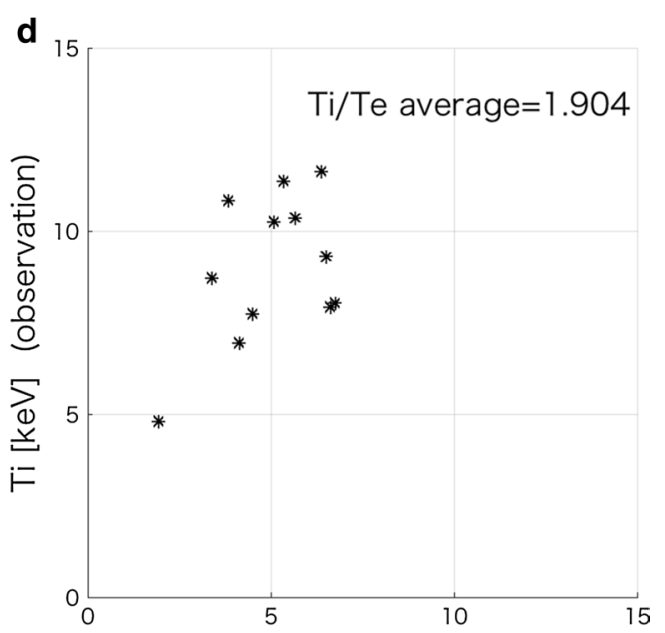

Te [keV] (observation)

Fig. 4 Extracted observational data at the time when $P_{e}$ peaked, leading to negative charging timing of pressure peak, and simulation data at the simulation time when $P_{\text {sim }}$ peaked within \pm 30 min from the time when $P_{e}$ peaked. Scatter plots of a $N_{e}$ versus $N_{\text {sim }}, \mathbf{b} T_{e}$ versus $T_{\text {sim }}$ c $P_{e}$ versus $P_{\text {sim, }}$ and $\mathbf{d}$ observed $T_{j}$ versus $T_{e}$
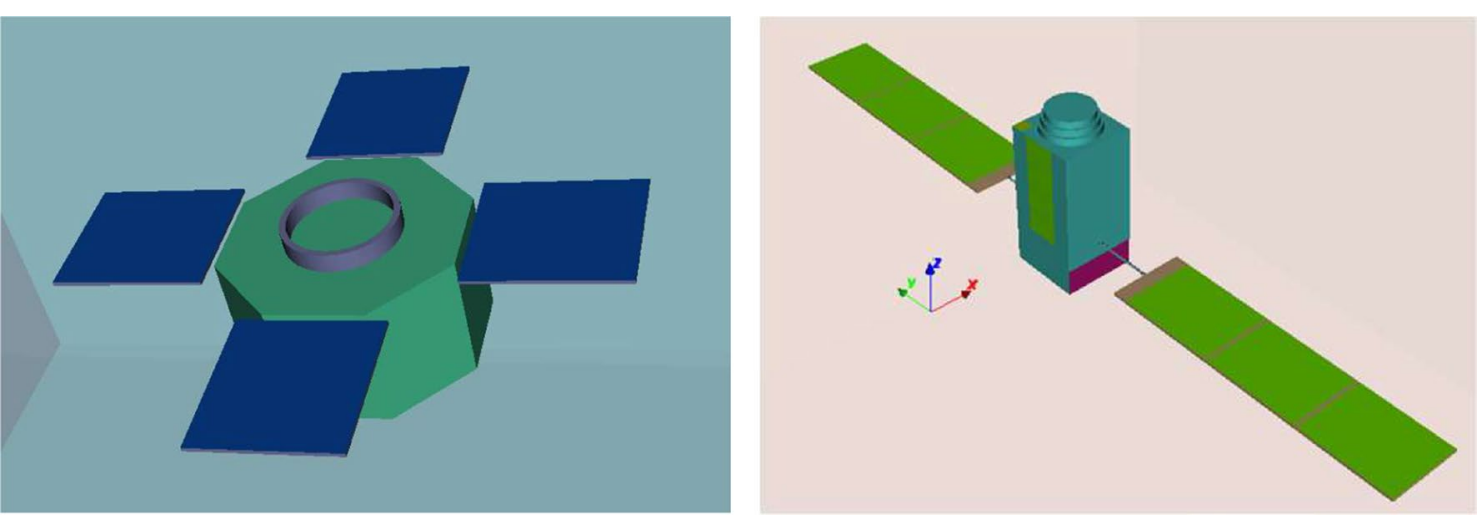

Fig. 5 Geometric models of Van Allen Probes (left) and Michibiki-1 satellite (right) 


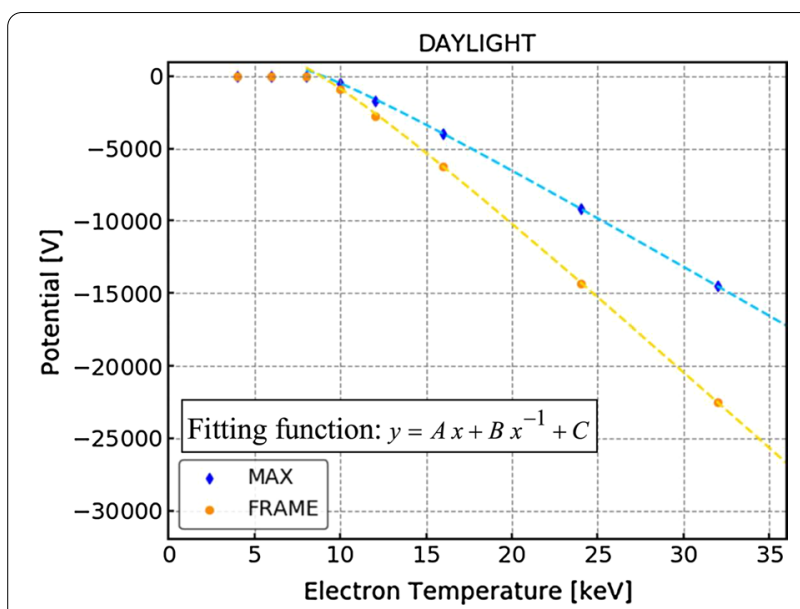

Fig. 6 Frame and maximum surface potentials estimated by SPIS with the geometric model of Michibiki-1 satellite under daylight condition

cc and $T_{i}=1.9^{*} T_{e}$ with $T_{e}$ as a single variable. Figure 6 shows an example of the frame (chassis ground) and maximum surface potentials of the Michibiki-1 satellite model in daylight as a function of $T_{e}$. In this case, several frame and maximum surface potentials are calculated for some values of $T_{e}$. A polynomial function is fitted for the results of the calculation, and we can obtain the empirical function of the surface charging potential from several precalculated results. With these functions, the frame and maximum surface potentials of the Michibiki-1 satellite can be quickly estimated using real-time simulation data. We also developed the empirical function for the surface potentials of the Van Allen Probes.

In the case of $N_{e}=N_{i}=2 / \mathrm{cc}$, the lines shown in Fig. 6 shift to the right by hundreds of $\mathrm{eV}$, but there is little change in their slope. The errors between the estimation method and the charging calculation results are usually a few percent, and even in bad cases are less than 10 percent when the calculations do not reach equilibrium even after $1 \mathrm{~h}$ or more of charging. The details of the estimation method are beyond the scope of this paper.

\section{Surface charging/ESD risk assessment system for GEO satellites}

The outline of the prototype surface charging/ESD risk assessment system is as follows. From the real-time simulation data per min, we extract the plasma environment data on the sphere of the 6.6 Earth radius $\left(R_{E}\right)$, which is identical to GEO, every $5 \mathrm{~min}$. The extracted data are stored in the system. The results of the prototype surface charging/ESD risk estimation system can be browsed with a web viewer. However, the web viewer is for internal use at present.

Since the explanation in the viewer is in Japanese, the content described in the viewer is shown as a schematic snapshot and a table. Figure 7 shows the schematic snapshot of the web viewer of the system, which was taken at 09:00 UT on July 05,2020 as an example. The left panel shows an overview of Earth's magnetosphere. The pressure and the bulk flow velocity distributions on the equatorial plane in the Solar Magnetic (SM) coordinate system are shown by color and arrows, respectively. The right panel shows the pressure distribution on the $6.6 \mathrm{R}_{\mathrm{E}}$ sphere in the geographic coordinate system. When we open the viewer, the latest condition is shown by default. At the top column, we can select the date and time of our interest. After selecting a specific time period, a 2-h movie from the selected start time can be played by controlling the bottom slider.

Because the photograph shown in Fig. 7 was taken at 09:00 UT, the local times of noon and midnight are at longitude $45^{\circ}$ and $225^{\circ}$, respectively, in the right panel. The high-pressure region around noon corresponds to the dayside cusps. On the other hand, another highpressure region is seen around midnight. It seems that the pressure enhancement around midnight is caused by magnetospheric activity on the nightside associated with a substorm. Real-time auroral electrojet indices show that the magnetosphere was in the expansion to recovery phases of a moderate substorm. The AL index was about $500 \mathrm{nT}$ at this time.

Table 1 lists examples of some parameters and estimated spacecraft potentials at the selected point shown as a pink cross in the right panel of Fig. 7. This table can also be browsed within the web viewer. When users mouse over the pink cross cursor on the display shown in the right panel of Fig. 7, the system returns the information of geographic latitude and longitude, daylight/eclipse flag, simulated pressure, density, and $\mathrm{T}_{\mathrm{e}}$ at the selected point. At the same time, the system calculates the spacecraft potentials using the empirical functions and plasma parameters described in the previous subsection. Here, the potentials include the following: the surface potential of a modeled scientific satellite, and the surface, frame, and differential potentials of a modeled commercial satellite, where the differential potential is the absolute value of the difference between the surface and frame potentials. The results are instantaneously shown in the table inside the web viewer.

Using this function, the satellite operators can select the position of their own satellite to estimate the absolute/differential potentials as an indication of the charging/ESD risk. Because the critical voltages for charging/ ESD risk are different in individual satellites owing to the 


\title{
Realtime Assessment of Satellite Surface Charging at Geosynchronous Region by Global Magnetosphere Simulation and Spacecraft Plasma Interaction Software
}

\author{
Data: 2020/07/06 \\ Time: 09:00
}

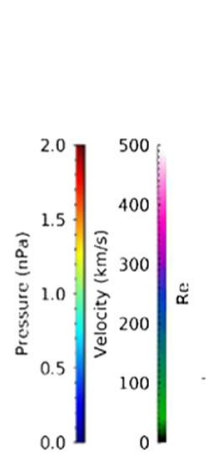

\begin{abstract}
Solar-Magnetic coordinate: Equator
\end{abstract}

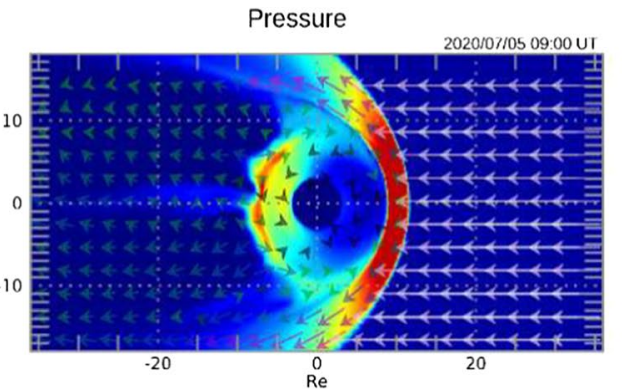

Geographic Coordinate :

Development view of $6.6 R_{E}$ sphere

Pressure

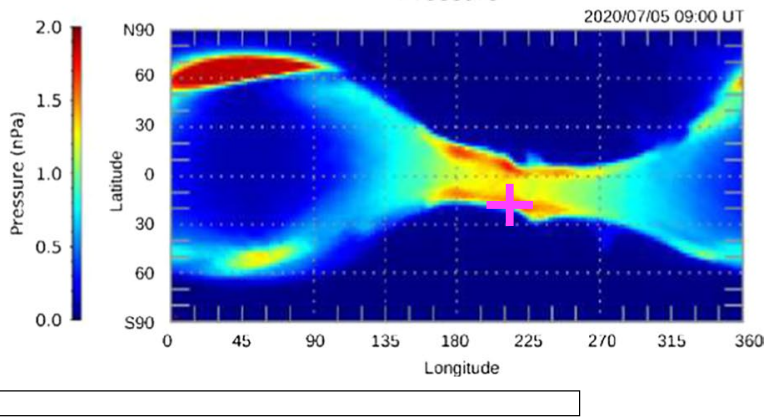

Time : 2020/07/05 09:00UT

Fig. 7 Schematic snapshot of the web viewer of the system taken at 09:00 UT on July 05, 2020. At present, the viewer is only in Japanese. The left panel shows the pressure and bulk flow velocity distributions on the equatorial plane in the SM coordinate system. The right panel shows the pressure distribution on the $6.6 R_{E}$ sphere in the geographic coordinate system. Specific date and time can be selected at the top column. A 2-h movie from the selected start time in the right panel can be played by controlling the bottom slider. The pink cross mark on the right panel of Fig. 7 can be moused over to examine the parameters and estimated satellite potentials. The result is instantaneously shown in the table inside the viewer. A schematic table is separately shown in Table 1

Table 1 Example of some parameters and estimated spacecraft potentials at the selected point shown as a pink cross in the right panel of Fig. 7

\begin{tabular}{lllll}
\hline Long. [deg.] & Lat. [deg.] & Density $[/ \mathrm{cc}]$ & Temperature $[\mathrm{keV}]$ & Pressure [nPa] \\
\hline 220.0 & -18.0 & 1.00 & 11.1 & 1.62 \\
\hline Eclipse $=1$ & Scientific satellite & Commercial satellite & & \\
Daylight $=\mathbf{0}$ & Surface potential $\Phi_{\text {sc }}[V]$ & Surface potential $\Phi_{\text {MAX }}[V]$ & Frame potential $\Phi_{\text {FRAME }}[V]$ & Differential potential $[V]$ \\
\hline 1 & -8700 & -5600 & -8100 & 2500 \\
\hline
\end{tabular}

This table is shown in the web viewer of the surface charging/ESD risk assessment system

differences in surface material and configuration, the satellite operators should use these potentials as a guide to understanding and evaluating the surface charging/ESD risk for their satellites.

To compare the satellite potential observed by LANL/ MPA and that estimated by our model, we calculated the potentials of a modeled scientific satellite using the estimated $T_{\text {sim-e }}$ of the February 15, 2006 event shown in Fig. 3. At the time when $P_{\text {sim }}$ peaked within $30 \mathrm{~min}$, $T_{\text {sim }}=2.26[\mathrm{keV}], P_{\text {sim }}=0.96[\mathrm{nPa}]$, and estimated $T_{\text {sim- }}$ $\mathrm{e}=6.98[\mathrm{keV}]$. (The observed $T_{e}$ was $6.624[\mathrm{keV}]$.) We obtained the satellite potential of -4032 [V] for the ecliptic condition. Although the estimated potential is lower than the potential observed by LANL/MPA, which reached about $-6000[\mathrm{~V}]$, this value is in relatively good agreement with the observation within a certain factor. This discrepancy is not significant because the shapes and materials of the modeled and LANL satellites are different from each other. In other words, even if the space environments are the same, the surface charging conditions are different for individual satellites.

\section{Internal charging part of SECURES}

To assess the risk of internal charging/ESD, anomalies of the Earth Sensor Assembly (ESA) onboard Kodama (DRTS: Data Relay Test Satellite) are examined as a test 
case. The internal charging of the ESA has been quantitatively assessed using MUSCAT with the ESA's structure and material model. The results of our assessment suggest that a detailed quantitative analysis is necessary to clarify internal charging/ESD. Thus, we have developed a risk assessment system of internal charging/ESD on the basis of a simple analysis method. Our achievements are described in detail in the following subsections.

\section{Satellite anomalies that occurred at ESA onboard Kodama (DRTS)}

Kodama (DRTS), which was launched on September 10, 2002, started routine operation on January 9, 2003. On March 23, 2003, a satellite anomaly occurred at the ESA onboard Kodama. The ESA shifted from the nominal system (ESA-A) to the redundant system (ESA-B). Subsequently, the same kind of anomaly occurred on April 2, May 27, and June 6, 2003. It was found that the ESA anomaly occurred during the period of increase in the ESA noise count. To identify the cause of the anomaly, the relationship between the ESA noise count and the high-energy electron flux observed by the Standard Dose Monitor (SDOM) onboard Kodama (Matsumoto et al. 2001) was examined. It was found that there is a relationship between the ESA noise count and Ch.3 of the differential electron flux (0.59-1.18 meV) observed by SDOM/ Kodama (Fig. 8). This relationship suggests that a possible cause of the ESA anomaly is internal charging/ESD. Using events of the satellite anomaly that occurred at the ESA onboard Kodama, we have developed an internal charging/ESD risk assessment system.
High-energy electron environment at GEO estimated using radiation belt model

To evaluate the energetic electron flux at the satellite location, we used the one-dimensional Fokker-Planck equation to describe the radial diffusion (Miyoshi et al. 2004), where $\mathrm{f}$ is the phase space density and $\mathrm{t}$ is time.

$$
\frac{\partial \mathrm{f}}{\partial \mathrm{t}}=L^{2} \frac{\partial}{\partial \mathrm{L}}\left(\frac{D_{\mathrm{LL}}}{L^{2}} \frac{\partial \mathrm{f}}{\partial \mathrm{L}}\right)-\sum_{i} \frac{f}{\tau_{\mathrm{i}}} .
$$

Here, $\mathrm{L}$ is the L-shell. We used the empirical radial diffusion coefficient $\left(D_{\mathrm{LL}}\right)$ formulated by Brautigam and Albert (2000). We consider the electromagnetic coefficients to be parameterized. The time step for calculation is $1 \mathrm{~h}$. The loss terms are described by the lifetimes due to Coulomb collisions $\left(\tau_{c}\right)$ and wave-particle interactions $\left(\tau_{\mathrm{wp}}\right)$ inside the plasmasphere. The lifetime $\tau_{c}$ is given by Wentworth et al. (1959). Three different wave sources inside the plasmasphere are included: plasmaspheric hiss, lightning whistler, and VLF transmitters. After the calculation of the phase space density $f$, we derived the differential flux $j(E, L, t)$, where $E$ is the electron energy. Considering the L-shell of Kodama, which varies during 1 day, we calculated the time variation of $j$ along the Kodama orbit. In this model, we used the condition of the empirical radiation belt model AE-8 (Vette 1991) as the initial condition.

\section{Internal charging estimation using MUSCAT}

MUSCAT is the charging analysis tool for satellite design. The development of MUSCAT started in 2004 and was completed in 2007 (Hosoda et al. 2008; Muranaka et al. 2008). MUSCAT has the analysis feature of surface charging, and the internal charging simulation function was added in 2010. In the internal charging analysis, the

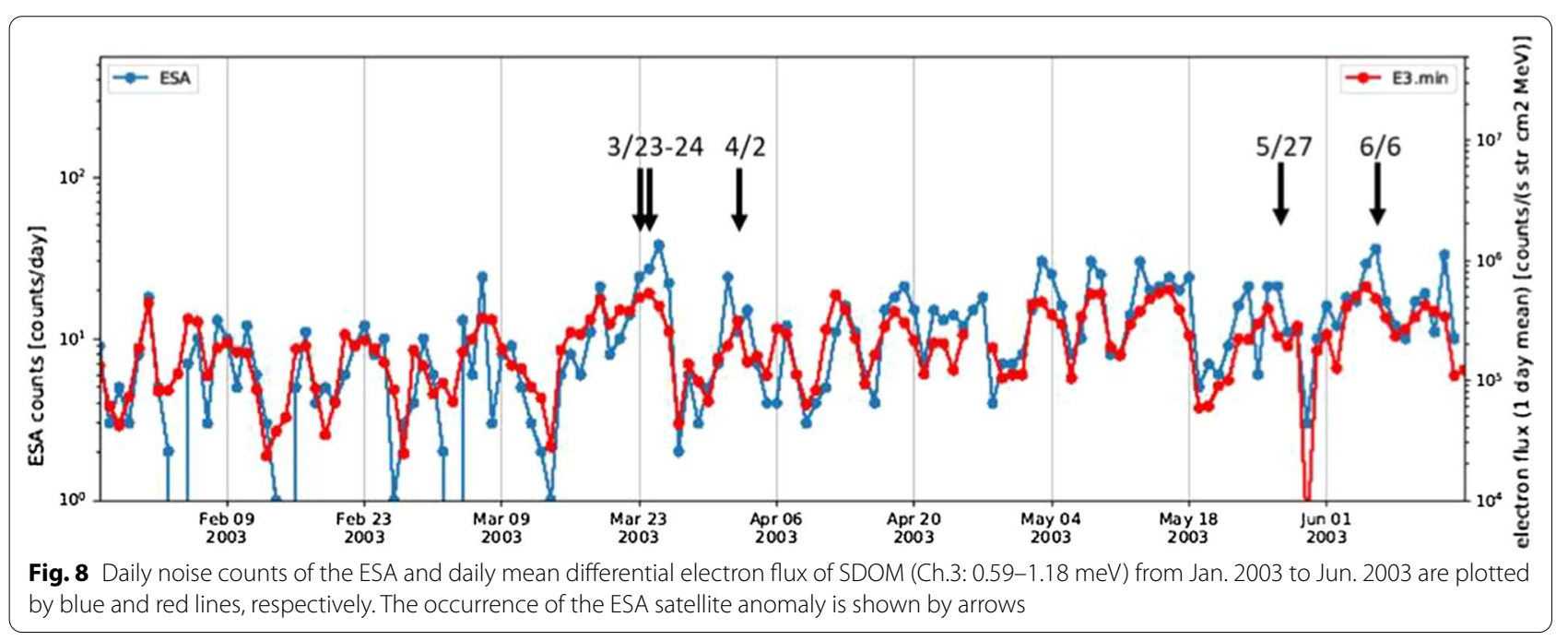


range of the high-energy electrons is calculated by the Monte Carlo method.

The structure and materials of the ESA were modeled using the MUSCAT graphical user interface. The external electron energy spectrum was estimated using the radiation belt model described in the previous subsection, and the electrostatic potential of the ESA was calculated using MUSCAT. It was found that the variation in the ESA electrostatic potential calculated using MUSCAT is very small compared with the threshold potential of the ESD. The structure around the ESA sensor has adequate thickness, which reduces the electron flux, so the charge accumulation is very small. This result suggests that the possibility of internal charging/ESD by other devices or cables, which are located under a thin shield, must be considered. An experimental study also suggested that noise produced by the ESD could propagate in panels and cables (Kinoda et al. 2018). A detailed quantitative analysis is necessary to clarify the internal charging/ESD of the ESA onboard Kodama, including the development of the detailed structure and material model inside the satellite. However, it is difficult to develop such a detailed model because data on the detailed structure and material are not usually disclosed by the satellite industry.

\section{Risk assessment system of internal charging/ESD}

The results discussed in the previous subsection suggest that the risk assessment of internal charging based on MUSCAT with the structure model of a specific part is unsuited to determining the cause of internal charging. Therefore, a risk assessment system of internal charging/ ESD has been developed on the basis of a simple analysis method introduced in NASA-HDBK-4002A (2017).

The procedure of our risk assessment system of internal charging/ESD is as follows. A simple structure model is used for our system. In this model, the thicknesses of the satellite shield $\left(d_{1}\right)$ and the target material $\left(d_{2}\right)$ are defined on request (Fig. 9). To estimate the range of incident electron energy into the target material $\left(d_{2}\right)$, the

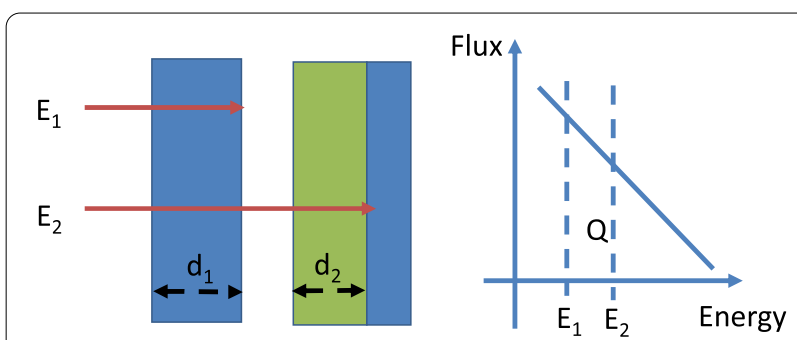

Fig. 9 (left) Simple structure model for internal charging. The thicknesses of the satellite shield $\left(d_{1}\right)$ and target material $\left(d_{2}\right)$ will be provided upon request. (right) Charge $(Q)$ of the $d_{2}$ region calculated from energy spectrum lower limit $\left(E_{1}\right)$ and higher limit $\left(E_{2}\right)$ of incident energy are converted from $d_{1}$ and $d_{1}+d_{2}$, respectively. The accumulated current inside the target material is calculated from the differential energy spectrum between $E_{1}$ and $E_{2}$ (Fig. 9). The differential energy spectrum is obtained from our radiation belt model described in a previous subsection. Alert levels for the risk of internal charging/ ESD are set to $0.1,0.3$, and $1.0 \mathrm{pA} / \mathrm{cm}^{2}$ on the basis of NASA-HDBK-4002A in accordance with the ESD Sensitivity Classification of the parts (MIL-STD-883G 2006). If the circuitry is classes 1 and 2 ESD sensitive, shield to levels of 0.1 and $0.3 \mathrm{pA} / \mathrm{cm}^{2}$, respectively. If the current exceeds $1 \mathrm{pA} / \mathrm{cm}^{2}$, internal ESD (IESD) may occur (NASA-HDBK-4002A). A flowchart of the alert system of internal charging is shown in Fig. 10.

An example of stacked plots for the risk assessment of ESA/Kodama internal charging/ESD using our system is shown in Fig. 11. In this calculation, $d_{1}$ and $d_{2}$ of aluminum are 0.22 and $0.15 \mathrm{~mm}$, respectively. This means that the higher and lower limits of the incident electron energy into the target material are 300 and $250 \mathrm{keV}$, respectively. Figure 11 shows that the correlation between the estimated current and anomalies of ESA/ Kodama is not high, but this system is capable of sending an alert of elevated risk to the user who set the thickness of the shield and the device in accordance with the ESD risk.

The basic functions of the risk assessment system of internal charging/ESD have been established. With this system, a customized alert for an individual user can be issued on the basis of the user's selection of the target material, which could be susceptible to internal charging/ ESD depending on the design of the operating satellite. If a user inputs the thicknesses of the satellite shield and the devices (target material) that may be susceptible to internal charging/ESD, the risk of satellite anomaly can be estimated on a routine basis using the prediction result of high-energy electron spectra obtained from our radiation belt model, and an alert can be issued in accordance with the excess of three threshold levels.

\section{Discussion and summary}

We have been developing SECURES for the risk assessment of surface charging/ESD and internal charging/ESD in GEO by combining a space environment model with a satellite charging model. We have confirmed the basic functions of SECURES as prototype products. In principle, these products could be used for practical purposes when we operate these products in near real time. To provide customized risk information for satellite operators, detailed data on the structures and materials of individual satellites are needed. However, as described in 


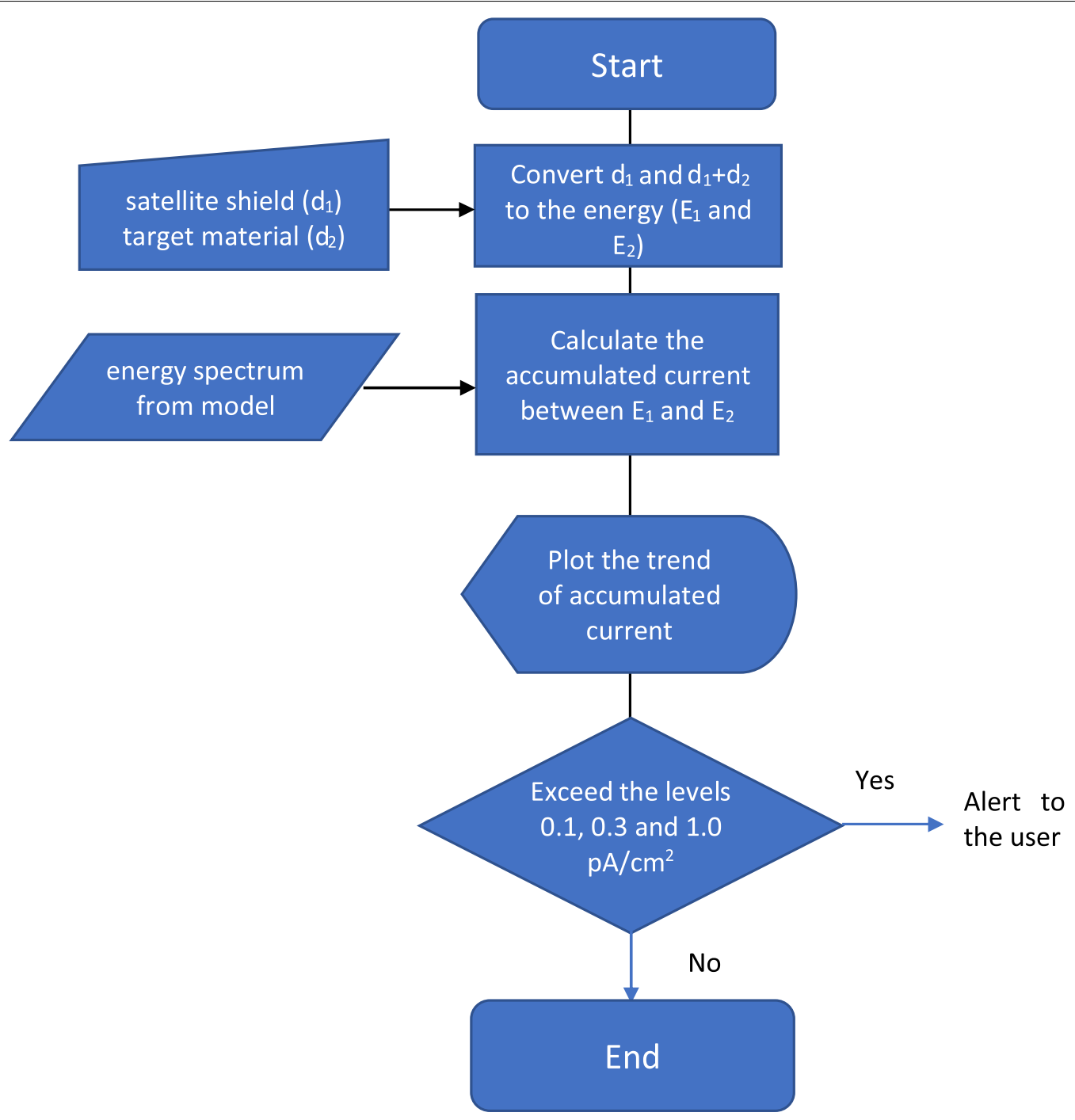

Fig. 10 Flowchart of the alert system of internal charging

the previous subsection, it is difficult to obtain such data because of nondisclosure from satellite industries. We need to promote further communication with the satellite industry to obtain detailed information on satellite structure and materials. Thus, we will start demonstrating our system on the basis of several sample models of satellites. Prototype products of SECURES will be provided to the public in the near future. These products will be useful for satellite operators and will improve the safety of satellite operation. We will also consider 


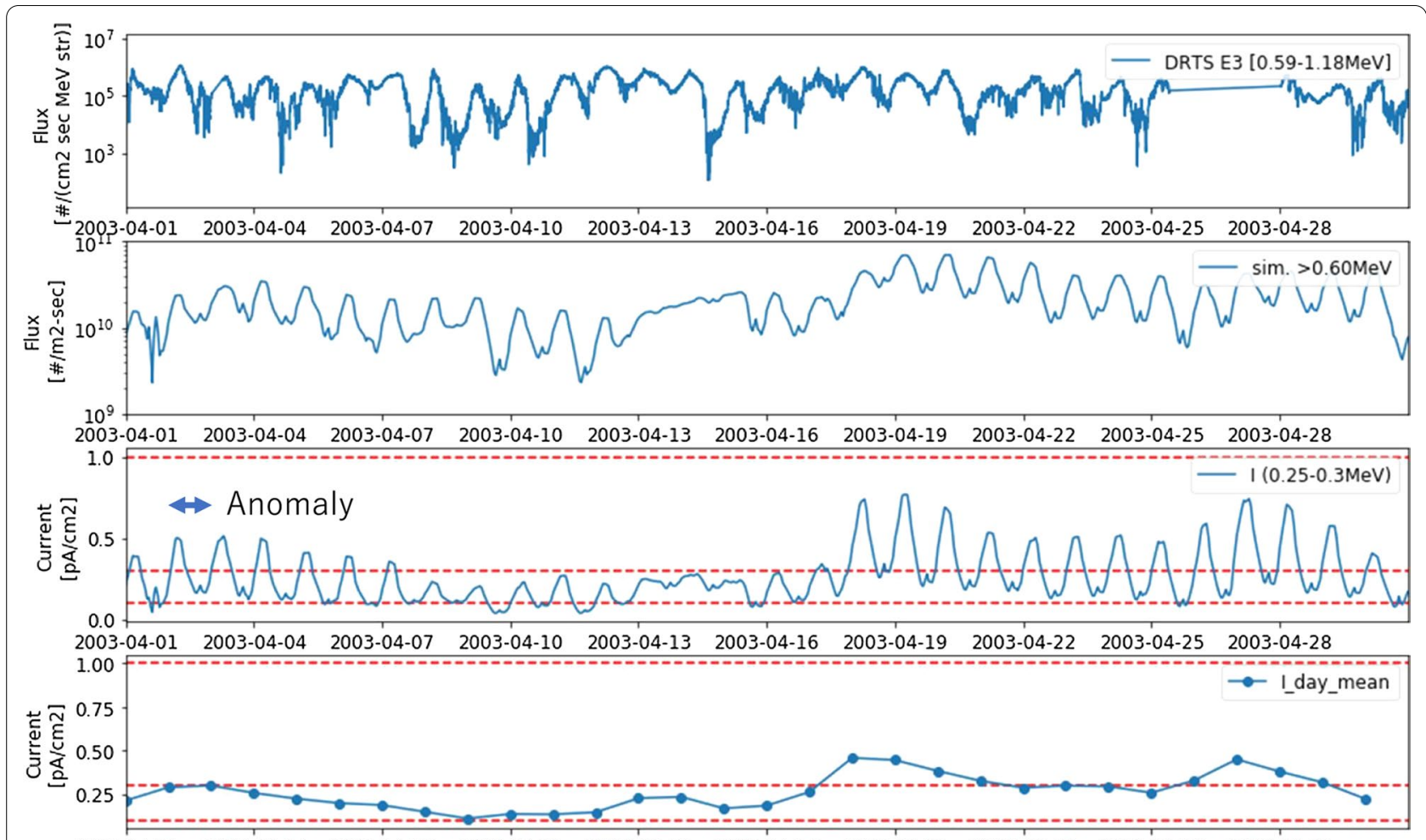

2003-04-01 2003-04-04 2003-04-07 $\quad 2003-04-10 \quad 2003-04-13 \quad 2003-04-16 \quad 2003-04-19 \quad 2003-04-22 \quad 2003-04-25 \quad 2003-04-28$

Fig. 11 Example of stacked plots for the risk assessment of internal charging/ESD. From top to bottom: differential electron flux observed by Ch.3 $(0.59-1.18 \mathrm{meV})$ of SDOM/Kodama, and differential electron flux estimated using the radiation belt model, current accumulated inside the target material $\left(d_{2}\right)$, and daily mean of current accumulated inside the target material $\left(d_{2}\right)$. The three dotted lines show the current levels of $0.1,0.3$, and $1.0 \mathrm{pA} / \mathrm{cm}^{2}$ in accordance with the ESD risk. The arrow shows the anomaly event of the ESA that occurred on April 2, 2003

expanding the target region from GEO to medium and low Earth orbits.

\begin{abstract}
Abbreviations
SECURES: Space environment customized risk estimation for satellites; MHD: Magnetohydrodynamics; ESD: Electrostatic discharge; SEP: Solar energetic particle; CPU: Central processing unit; UN/COPUOUS: United Nations/Committee on the Peaceful Uses of Outer Space; PSTEP: Project for Solar-Terrestrial Environment Prediction; SPIS: Spacecraft plasma interaction software; MUSCAT : Multi-utility spacecraft charging analysis tool; GEO: Geostationary earth orbit; MPI: Message passing interface; REPPU: REProduce Plasma Universe; NICT: National Institute of Information and Communications Technology; NOAA SWPC: National Oceanic and Atmospheric Administration's Space Weather Prediction Center; MPA: Magnetospheric plasma analyzer; LANL: Los Alamos National Laboratory; CDAWeb: Coordinated data analysis web; CPCP: Cross polar cap potential; $\mathrm{R}_{\mathrm{E}}$ : Earth radius; SM: Solar magnetic; ESA: Earth sensor assembly; DRTS: Data relay test satellite.
\end{abstract}

\section{Acknowledgements}

We thank K. Sakaguchi for helpful discussions. Real-time solar wind data at the L1 point were provided by NOAA/SWPC. Observed plasma moment data of LANL/MPA were downloaded from NASA/CDAWeb. The real-time auroral electrojet indices were provided by the World Data Center for Geomagnetism, Kyoto.

\section{Authors' contributions}

TN led the development of SECURES and edited the paper. AN and YK improved the global magnetosphere MHD model and prototype surface charging/ESD risk assessment system. MN developed the empirical functions of surface charging estimation by SPIS. KK examined the internal charging risk by MUSCAT and developed the prototype internal charging/ESD risk assessment system based on a simple analysis method. YM developed the radiation belt model. HM provided satellite anomaly data of ESA/Kodama and supervised the project of SECURES. All the authors read and approved the final manuscript.

\section{Funding}

Part of this study is supported by JSPS KAKENHI 15H05813 and 15H05815, Project for Solar-Terrestrial Environment Prediction (PSTEP), and by "Promotion of observation and analysis of radio wave propagation," commissioned research of the Ministry of Internal Affairs and Communications, Japan.

\section{Availability of data and materials}

The real-time solar wind data at the L1 point provided by NOAA/SWPC are available at https://www.swpc.noaa.gov/products/real-time-solar-wind. LANL/ MPA data provided by NASA/CDAWeb are available at https://cdaweb.gsfc. nasa.gov/index.html. The data obtained from global magnetosphere MHD simulation can be obtained from A. Nakamizo upon request. SPIS is available at http://dev.spis.org/projects/spine/home/spis. The geometric model of Van Allen Probes can be obtained from M. Nakamura upon request. The geometric model of the Michibiki-1 satellite cannot be shared because the model includes nondisclosure information. The data obtained from empirical models of estimating the potential of surface charging can be obtained from M. Nakamura upon request. The real-time auroral electrojet indices provided by the World Data Center for Geomagnetism, Kyoto, are available at http:// wdc.kugi.kyoto-u.ac.jp/ae_realtime/index.html. SDOM/Kodama data are available at http://sees.tksc.jaxa.jp/fw/dfw/SEES/index.html. ESA/Kodama anomaly data cannot be shared because of the data policy of JAXA. The data obtained from the radiation belt model can be obtained from Y. Miyoshi upon 
request. MUSCAT Space Engineering Co., Ltd. (MUSE: http://astro-muse.com/) sells MUSCAT software and provides related support. Currently, services are only provided domestically. The data obtained from a simple internal charging model can be obtained from K. Koga upon request.

\section{Ethics approval and consent to participate}

Not applicable.

\section{Consent for publication}

Not applicable.

\section{Competing interests}

The authors declare that they have no competing interests.

\section{Author details}

${ }^{1}$ National Institute of Information and Communications Technology, 2-1-3 Katahira, Aoba-ku, Sendai, Miyagi 980-0812, Japan. ${ }^{2}$ National Institute of Information and Communications Technology, 4-2-1 Nukui-kita, Koganei, Tokyo 184-8795, Japan. ${ }^{3}$ Osaka Prefecture University, 1-1 Gakuen-cho, Naka-ku, Sakai, Osaka 599-8531, Japan. ${ }^{4}$ Japan Aerospace Exploration Agency, Sengen 2-1-1, Tsukuba, Ibaraki 305-8505, Japan. ${ }^{5}$ Nagoya University, Nagoya 464-8601, Japan.

Received: 22 August 2020 Accepted: 4 January 2021

Published online: 25 January 2021

\section{References}

Brautigam DH, Albert JM (2000) Radial diffusion analysis of outer radiation belt electrons during the October 9, 1990, magnetic storm. J Geophys Res 105:291-309. https://doi.org/10.1029/1999JA900344

Ferguson DC, Worden SP, Hastings DE (2015) The space weather threat to situational awareness, communications, and positioning systems. IEEE Trans Plasma Sci 43(9):3086-3098. https://doi.org/10.1109/TPS.2015.2412775

Hosoda S, Muranaka T, Kuinaka H, Kim J, Hatta S, Kurahara N, Cho M, Ueda HO, Koga K, Goka T (2008) Laboratory experiments for code validation of multiutility spacecraft charging analysis tool (MUSCAT). IEEE Trans Plasma Sci 36(5):2350-2359. https://doi.org/10.1109/TPS.2008.2003973

Inaba N, Matsumoto A, Hase H, Kogure S, Sawabe M, Terada K (2009) Design concept of Quasi Zenith Satellite System. Acta Astronaut 65:1068-1075. https://doi.org/10.1016/j.actaastro.2009.03.068

Kinoda H, Niki Y, Sasaki Y, Nakamoto F, Cho M, Toyoda K (2018) Noise propagation due to electrostatic charging/discharging and its effect. In: Proceedings of the 15th space environment symposium, p. 109-116 (in Japanese).

Koons HC, Mazur JE, Selesnick RS, Blake JB, Fennell JF, Roeder JL, Anderson PC (1999) The impact of the space environment on space systems. SMCTR-00-10, Aerospace report NO. TR-99(1670)0-1.

Kubota Y, Nagatsuma T, Den M, Tanaka T, Fujita S (2017) Polar cap potential saturation during the Bastille day storm event using global MHD simulation. J Geophys Res 122:4398-4409. https://doi.org/10.1002/2016JA023851

Kubota Y, Nagatsuma T, Nakamizo A, Sakaguchi K, Den M, Matsumoto H, Higashio N, Tanaka T (2019) Comparison of magnetospheric magnetic field variations at quasi-zenith orbit based on Michibiki observation and REPPU global MHD simulation. IEEE Trans Plasma Sci 47(8):3937-3941. https://doi.org/10.1109/TPS.2019.2910301

Matsumoto H, Koshiishi H, Goka T, Kimoto Y, Green BD, Galica GE, Nakamura T, Abe T, Badono S, Murata S, Sullivan JD (2001) Compact, lightweight spectrometer for energetic particles. IEEE Trans Nuclear Sci 48(6):2043-2049. https://doi.org/10.1109/23.983170

MIL-STD-883G (2006) Test method standard microcircuits, department of defense.
Miyoshi YS, Jordanova VK, Morioka A, Evans DS (2004) Solar cycle variations of the electron radiation belts: observations and radial diffusion simulation. Space Weather 2:S 10S02. https://doi.org/10.1029/2004SW000070

Muranaka T, Hosoda S, Kim JH, Hatta S, Ikeda K, Hamanaga T, Cho M, Usui H, Ueda HO, Koga K, Goka T (2008) Development of multi-utility spacecraft charging analysis tool (MUSCAT). IEEE Trans Plasma Sci 36(5):2336-2349. https://doi.org/10.1109/TPS.2008.2003974

Nagatsuma T (2017) Toward implementing practical space environment prediction for safety and security of satellite operation. Aeronaut Space Sci Jpn 65:96-99. https://doi.org/10.14822/kjsass.65.4_96 (in Japanese)

Nagatsuma T, Matsumoto H, Kubota Y, Nakamizo A, Koga K (2018) Comparison between surface charging event from MICHIBIKI (QZS) satellite and space environment data from global MHD simulation. Trans Jpn Soc Aeronaut Space Sci Aerospace Tech Jpn 16(2):157-160. https://doi.org/10.2322/ tastj.16.157

Nakamizo A, Tanaka T, Kubo Y, Kamei S, Shimazu H, Shinagawa H (2009) Development of the 3-D MHD model of the solar corona-solar wind combining system. J Geophys Res 114:A07109. https://doi.org/10.1029/2008JA0138 44

Nakamura M (2012) Forecast of the plasma environment in the geostationary orbit using the magnetospheric simulation. J Plasma Fusion Res 88:83-86 (in Japanese)

NASA-HDBK-4002A (2017) Mitigating In-Space Charging Effects-A Guideline, Nasa Technical Handbook.

O'Brien TP, Mazur JE, Fennell JF (2013) The priority mismatch between space science and satellite operations. Space Weather 11:49. https://doi. org/10.1002/swe20028

Roussel JF, Rogier F, Dufour G, Mateo-Velez JC, Forest J, Hilgers A, Rodgers D, Girard L, Payan D (2008) SPIS Open-source code: methods, capabilities, achievements, and prospects. IEEE Trans Plasma Sci 36(5):2360-2368. https://doi.org/10.1109/TPS.2008.2002327

Stratton JM, Harvey RJ, Heyler GA (2013) Mission overview for the radiation belt storm probes mission. Space Sci Rev 179:29-57. https://doi. org/10.1007/s11214-012-9933-x

Tanaka T (1994) Finite volume TVD scheme on an unstructured grid system for three-dimensional MHD simulation of inhomogeneous systems including strong background potential fields. J Comput Phys 111:381-389. https://doi.org/10.1006/jcph.1994.1071

Tanaka T (2015) Substorm auroral dynamics reproduced by advanced global magnetosphere-ionosphere (M-I) coupling simulation. In: Zhang Y (ed) Auroral dynamics and space weather, Geophys Monogr Ser. vol 215 AGU, Washington DC. p.177 https://doi.org/10.1002/9781118978719.ch13

Tanaka T, Nakamizo A, Yoshikawa A, Fujita S, Shinagawa H, Shimazu H, Kikuchi T, Hashimoto KK (2010) Substorm convection and current system deduced from the global simulation. J Geophys Res 115:A05220. https:// doi.org/10.1029/2009JA014676

Troshichev O, Hayakawa H, Matsuoka A, Mukai T, Tsuruda K (1996) Cross polar cap diameter and voltage as a function of $\mathrm{PC}$ index and interplanetary quantities. J Geophys Res 101:13429-13435. https://doi. org/10.1029/95JA03672

Vette II (1991) The NASA/National space science data center trapped radiation environment model program (1964-1991), NSSDC Report 91-29. Greenbelt, MD

UN/COPUOS (2019) Guidelines for the long-term sustainability of outer space activities, A/AC. 105/C.1/L.366

Wentworth RC, MacDonald WM, Singer SF (1959) Lifetimes of trapped radiation belt particles determined by Coulomb scattering. Phys Fluids 2:499-509

\section{Publisher's Note}

Springer Nature remains neutral with regard to jurisdictional claims in published maps and institutional affiliations. 